Research Article

\title{
Preparation and Characterisation of Polypyrrole-Iron Oxyhydroxide Nanocomposite as Sensing Material
}

\author{
Farah Nabila Diauudin, ${ }^{1}$ Siti Aminah Mohd Noor $\left(\mathbb{D},{ }^{2}\right.$ Jahwarhar Izuan Abdul Rashid (iD), \\ Victor Feizal Knight $\left(\mathbb{D},{ }^{3}\right.$ Wan Md Zin Wan Yunus $\left(\mathbb{D},{ }^{4}\right.$ Keat Khim Ong, ${ }^{3}$ \\ Noor Azilah Mohd Kasim, ${ }^{3}$ Safura Taufik, ${ }^{2}$ Alinda Samsuri, ${ }^{2}$ Intan Juliana Shamsudin, ${ }^{2}$ \\ and Wahhida Latip ${ }^{5}$
}

${ }^{1}$ Faculty of Defence Science and Technology, National Defence University of Malaysia, Sungai Besi Camp,

Kuala Lumpur 57000, Malaysia

${ }^{2}$ Centre for Defence Foundation Studies, National Defence University of Malaysia, Sungai Besi Camp, Kuala Lumpur 57000, Malaysia

${ }^{3}$ Research Centre for Chemical Defence, National Defence University of Malaysia, Sungai Besi Camp, Kuala Lumpur 57000, Malaysia

${ }^{4}$ Centre for Tropicalisation, National Defence University of Malaysia, Sungai Besi Camp, Kuala Lumpur 57000, Malaysia

${ }^{5}$ Enzyme and Microbial Technology Research Center, Faculty Biotechnology and Biomolecular Science,

Universiti Putra Malaysia, Jalan Universiti 1, Serdang, Seri Kembangan 43400, Selangor, Malaysia

Correspondence should be addressed to Siti Aminah Mohd Noor; s.aminah@upnm.edu.my

Received 4 August 2020; Revised 6 November 2020; Accepted 25 November 2020; Published 14 December 2020

Academic Editor: Yue Hou

Copyright ( 92020 Farah Nabila Diauudin et al. This is an open access article distributed under the Creative Commons Attribution License, which permits unrestricted use, distribution, and reproduction in any medium, provided the original work is properly cited.

\begin{abstract}
Polypyrrole (PPy) has been widely used as a sensing material in many studies as PPy gives out noticeable reactions towards varieties of vapours of acids, bases, alcohols, alkanes, and chemical warfare agent (CWA) simulants. However, due to dispersion and mechanical properties limitation, PPy in nanoparticles (PPy NPs) has been introduced. The combinations between PPy NPs and nanosized metal oxide have shown promising improvement in the sensitivity of a sensor due to the larger total surface area of detection. In this study, the potential of PPy NPs and polypyrrole-iron oxyhydroxide (PFFs) nanocomposite as sensing materials in detecting the CWA simulant, dimethyl methylphosphonate (DMMP), is investigated. PPy NPs and modified PFFs nanocomposite were successfully synthesised using chemical oxidative polymerisation. The formation of PPy NPs and the effect of iron-oxyhydroxide $(\mathrm{FeOOH})$ on the chemical interaction, morphology, and size of PPy NPs were investigated using attenuated total reflection-Fourier transform infrared (ATR-FTIR), field emission scanning electron microscopy (FESEM), and transmission electron microscopy (TEM). ATR-FTIR analysis showed that the modified PFFs nanocomposite was successfully produced through the presence of a sharp-OH peak. The size of the prepared PPy NPs and PFFs nanocomposite was observed in the range of 50 to $70 \mathrm{~nm}$ and 110 to $160 \mathrm{~nm}$, respectively, through TEM analysis. The electrochemical behaviour of PPy NPs and PFFs nanocomposite towards DMMP was investigated using cyclic voltammetry $(\mathrm{CV})$ where, in the presence of DMMP, PPy NPs and PFFs nanocomposite showed $24 \%$ and $21 \%$ current signal reduction, respectively. This suggests that both PPy NPs and PFFs nanocomposite are able to discriminate an electrical signal both with and without the presence of DMMP.
\end{abstract}

\section{Introduction}

Polypyrrole (PPy) is one of the conducting polymers (CPs) that exhibit optical and electrical properties of both semiconductors and metals and are known as a distinctive group of organic materials $[1,2]$. Poly(3,4-ethylene dioxythiophene) (PEDOT), polythiophene, polyaniline, and polyacetylene are the other examples of commonly used CPs 
described in the research [3]. These polymers are commonly synthesised through chemical or electrochemical oxidation of monomers using oxidant agents through the conjugated bond system with the polymer backbone being rendered conductive [4]. CP has attracted much attention among researchers due to its inexpensive production, thermal stability, simple synthesis process, and presence of adequate electrical conductivity [5]. Among CPs, PPy is one of the most promising of these polymers because of its remarkable chemical stability and electrical conductivity [6]. However, one of the limitations is that it is unable to be well dispersed during synthesis due to its strong interchain reactions and is somewhat lacking in mechanical properties that affect fabrication processes $[7,8]$. Therefore, PPy in nanoparticle sizes has been explored to improve these limitations as PPy NPs have a much larger surface area and have a porous form [9].

This PPy NP can be synthesised using different oxidants such as ammonium persulfate $\left(\mathrm{N}_{2} \mathrm{H}_{8} \mathrm{~S}_{2} \mathrm{O}_{8}\right)$, iron (III) sulfate $\left(\mathrm{Fe}_{2}\left(\mathrm{SO}_{4}\right)_{3}\right)$, and iron (III) chloride $\left(\mathrm{FeCl}_{3}\right)[10,11]$. As described by Rao et al. [12], $\mathrm{FeCl}_{3}$ is commonly chosen as an oxidant due to its stabilisation oxidation process with the pyrrole monomer as is shown in equation (1), and it is able to give higher conductivity properties compared to other oxidants [13]. Then, to further enhance the synthesis, surfactants also play an important role in the polymerisation process by improving the physical properties of polymers such as their solubility in solvents, stability, and conductivity [14]. Sodium dodecylsulfate (SDS), polystyrene sulfonate, dodecylbenzenesulfonic acid, and sodium dodecylbenzenesulfonate (SDBS) are examples of surfactants used in the polymerisation processes of polymers $[15,16]$. As reported by Xing and Zhao [17], SDBS has been commonly utilised as a surfactant compared to any others as it can enhance the stabilisation of PPy NPs in terms of its dispersion state.

$$
\mathrm{Fe}^{3+}+1 \mathrm{e}^{-} \longrightarrow \mathrm{Fe}^{2+} .
$$

Over the last few years, PPy NP has been used as a sensing material for the detection of the analyte DMMP which is known as a simulant of chemical warfare nerve agents. This simulant is a chemical that is able to mimic both the physical and chemical properties of nerve agents without its toxic effects [18]. Diethyl chlorothiophosphate (DCTP), methylphosphonic dichloride (MPDC), diisopropylfluorophosphate (DFP), and dimethyl methylphosphonate (DMMP) are several types of simulants that are used to substitute actual nerve agents (CWAs) for research and training because of safety issues with the use of actual nerve agents for these purposes [19]. DMMP is a widely utilised simulant for CWAs because of its nontoxicity and organophosphorus compound elemental composition that imitates many nerve agents $[20,21]$.

As a result of its unique properties, PPy NPs have been employed as sensing materials for various detection methods against chemicals in pesticides, air pollutants, and acidic gases [22]. In order to improve its sensing performance, surface modification of PPy NPs is needed because this would be able to increase the sensitivity of the sensor towards the analyte. In previous works, Kwon et al. [23] have shown that the introduction of carboxylic groups on the surface of PPy nanotube transistors has resulted in increased sensitivity towards DMMP via intermolecular hydrogen bonding while a combination of PPy with copper phthalocyanine was reported to be able to detect DMMP gas simulants through its high electron-donating nature [24]. Furthermore, Das and Roy [25] claimed that PPy NPs and titanium dioxide $\left(\mathrm{TiO}_{2}\right)$ increased the exposure of hydrogen gas due to the enhanced surface area of the composite and the increased diffusion pathway of the hydrogen molecule. Malook et al. [26] also have synthesised polypyrrole-bimetallic oxide composites ( $\mathrm{PPy}-\mathrm{V} 2 \mathrm{O} 5-\mathrm{MnO} 2$ ) and found that the resistance of the sensor was increased when exposed to ammonia gas due to doping of PPy during the synthesis of lead composites that contained positive charge carrier. Other work by Šetka et al. [27] has demonstrated that zinc oxide-based polypyrrole (ZnO-based PPy) nanocomposites were able to detect ammonia better as compared to tin (IV) oxide $\left(\mathrm{SnO}_{2}\right)$-based PPy. This is due to the attributes that $\mathrm{Zn}^{2+}$ possesses in which it is redox-active, thus influencing the charge density and conductivity of the nanocomposites. Recently, FeOOH NPs have shown pronounced results in detecting DMMP when the FeOOH NPs were attached to the PPy structure (by replacing $\mathrm{H}$ in the $\mathrm{N}$ atom) [28].

In this present study, PPy NPs and modified PFFs nanocomposite have been synthesised using chemical oxidative polymerisation. The chemical interaction and morphology of the synthesised PPy NPs and PFFs nanocomposite were then characterised using Fourier transform infrared (FTIR), field emission scanning electron microscopy (FESEM), and transmission electron microscopy (TEM). The potential of the synthesised PPy NPs and PFFs nanocomposite as sensing materials was then investigated using cyclic voltammetry $(\mathrm{CV})$.

\section{Methodology}

2.1. Materials. Pyrrole reagent grade (98\%), sodium dodecylbenzenesulfonate (SDBS), sodium hydroxide $(\mathrm{NaOH})$, iron (III) chloride (97\%), and gold nanoparticle suspension were acquired from Sigma-Aldrich. Ethanol (95\%) was purchased from HighChem, Hamburg. All of these chemicals were used as received.

2.2. Polypyrrole Polymerisation Process. Firstly, 40 wt $\%$ of SDBS was stirred magnetically in $40 \mathrm{~mL}$ of distilled water at room temperature. After that, 18.5 wt $\%$ with $14.9 \mathrm{mmol}$ of pyrrole monomer was added to the surfactant solution dropwise while $40.1 \mathrm{wt} \%$ ferric chloride $\left(\mathrm{FeCl}_{3}\right)$ that has been dissolved in distilled water was then added into the mixture of surfactant/ pyrrole. The chemical polymerisation proceeded for $2 \mathrm{~h}$ at room temperature. In order to improve the distribution of nanoparticles, a sonication method was introduced into the preparation of PPy NPs. The mixture was sonicated for an hour and three hours to obtain well-dispersed nanoparticles in the solution before being washed with an excess of methyl alcohol and finally being dried in a vacuum at room temperature. Figure 1 shows the schematic diagram for the PPy NPs polymerisation process. 


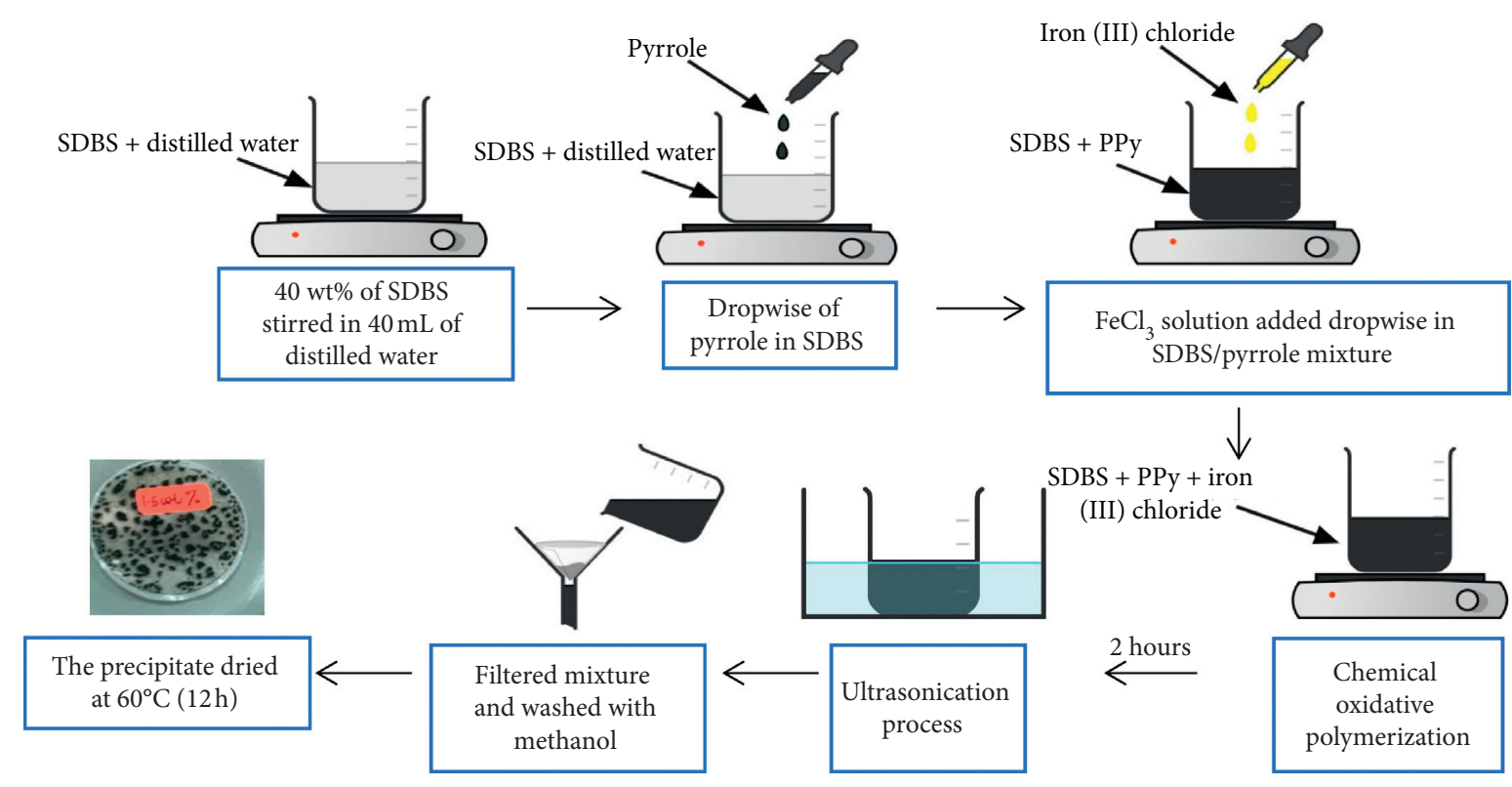

FIgURE 1: Schematic diagram for the PPy NPs polymerisation process.

2.3. Fabrication of Polypyrrole Nanoparticles-Iron Oxyhydroxide (PFFs). The obtained PPy NPs were then mixed for two hours with 10 wt\% of $\mathrm{FeCl}_{3}$ into two separate beakers with the same process. Then, both of the mixtures were dropped into sodium hydroxide $(\mathrm{NaOH})$ solution with two different concentrations (1.9 and 3.7 wt\%) and were left to mix for an hour. These mixtures were then separated into two different weight percentages of $\mathrm{FeCl}_{3}(5$ $w t \%$ and $10 \mathrm{wt} \%)$. In addition, each mixture was magnetically stirred for two hours to ensure all of its components were completely mixed, followed by sonication for an hour at room temperature. Finally, ethanol was used to wash the obtained precipitate several times before being dried inside a vacuum oven at $60^{\circ} \mathrm{C}$ for 12 hours. The schematic diagram for the preparation of PFFs nanocomposite is shown in Figure 2.

\subsection{Characterisations}

2.4.1. Fourier Transform Infrared Spectroscopy (FTIR). The polymerisation process of pyrrole to PPy NPs and the combination of PFFs nanocomposite were analysed to obtain the different functional group bands of the prepared samples. The FTIR used was a PerkinElmer Spectrometer Frontier FTIR with a frequency range of $650-4000 \mathrm{~cm}^{-1}$, and the transmission bands of each were scanned at a spectrum scan rate of $4 \mathrm{~cm}^{-1}$. Baseline correction was done for all the prepared samples.

2.4.2. Field Emission Scanning Electron Microscopy (FESEM). The surface condition or morphology of the PPy NPs and the PFFs nanocomposite was observed using a field emission scanning electron microscope (FESEM) (Zeiss, Gemini500 SEM) with 10,000x magnification.
2.4.3. Transmission Electron Microscopy (TEM). TEM analysis was conducted using a FEI Talos L120C with a resolution of $120 \mathrm{kV}$ and $120 \mathrm{~K}$ magnification and used to obtain the sizes of the PPy NPs and PFFs nanocomposite since the sizes of these materials play an important role in this experiment with respect to conductivity and total surface area of absorption.

2.4.4. Cyclic Voltammetry (CV). Electrochemical analysis of the PPy NPs and PFFs nanocomposite was performed using the $\mathrm{CV}$ technique. A screen-printed carbon electrode (SPCE) was used as the electrode. The SPCE is typically composed of a carbon layer as the working and counter electrode, whereas a silver layer is used as the reference electrode that is connected to a Metrohm Autolab B.V (PGSTAT204) potentiostat. $10 \mu \mathrm{L}$ gold nanoparticle suspension, PPy NPs, and PFFs nanocomposite were drop cast onto the SPCE to detect $10 \mu \mathrm{L}$ of DMMP. The scan rate used was $50 \mathrm{mVS}^{-1}$ for eight cycles. All measurements were carried out at room temperature. Figure 3 depicts the setup of the electrochemical sensing system using a screen-printed electrode.

\section{Results and Discussion}

3.1. Fourier Transform Infrared Spectroscopy of Pyrrole Monomer and Nanoparticle Polypyrrole. FTIR analysis was conducted to study the formation of PPy NPs from its monomer. As shown in Figure 4, the pyrrole monomer gives out a sharp peak of $\mathrm{N}-\mathrm{H}$ stretching vibration bands at $3397 \mathrm{~cm}^{-1}$ while the PPy NPs showed broad main absorption peaks at $3217 \mathrm{~cm}^{-1}$ due to the stacking of monomer rings of $\mathrm{N}-\mathrm{H}$ groups, which indicated that polymerisation had occurred [29, 30]. An obvious peak of symmetric and asymmetric C-H stretching vibrations of saturated hydrocarbons of the PPy was shown at $2887 \mathrm{~cm}^{-1}$ compared to a small peak of 


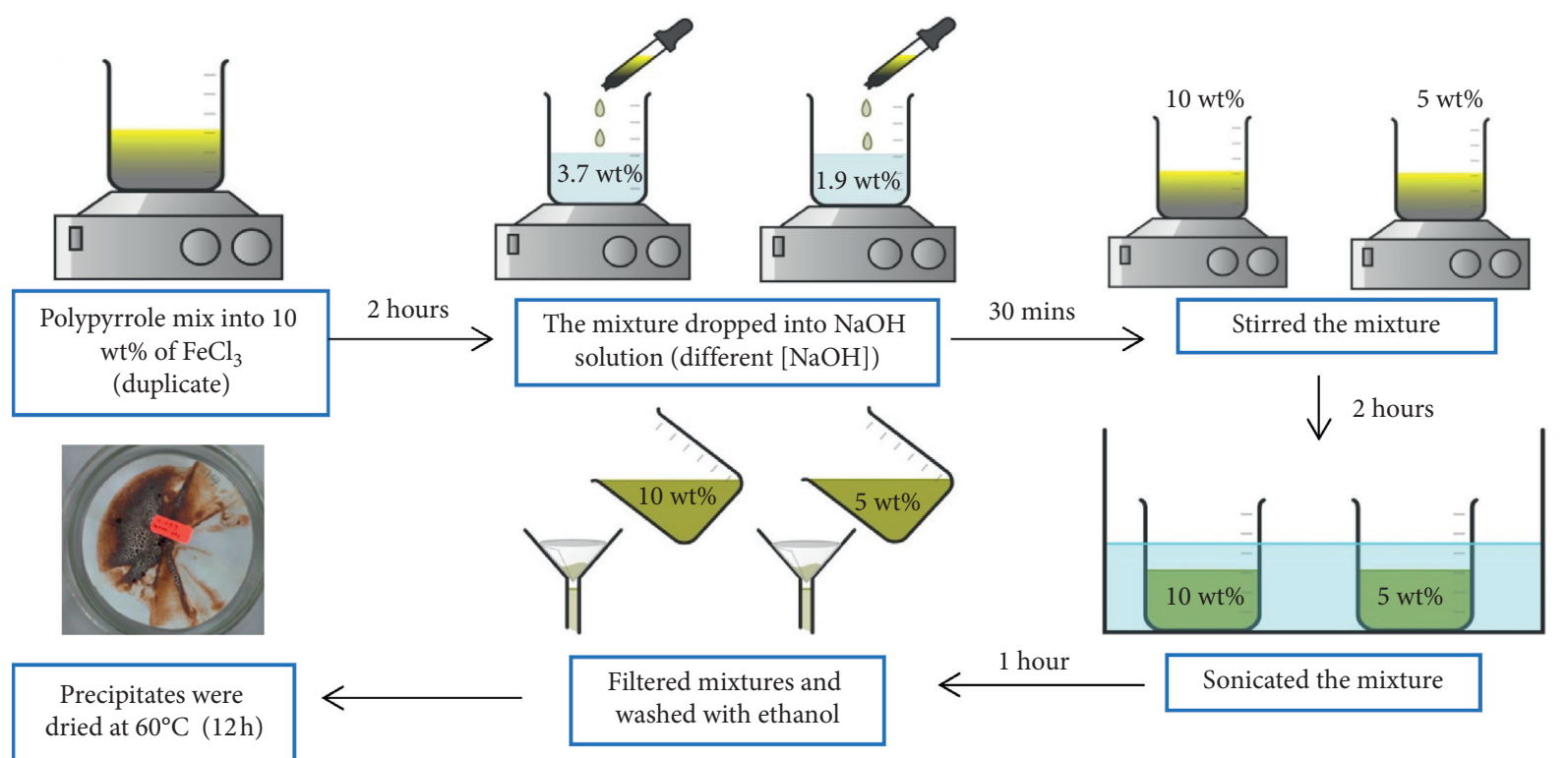

FIGURE 2: Schematic diagram for the preparation of PFFs nanocomposite.

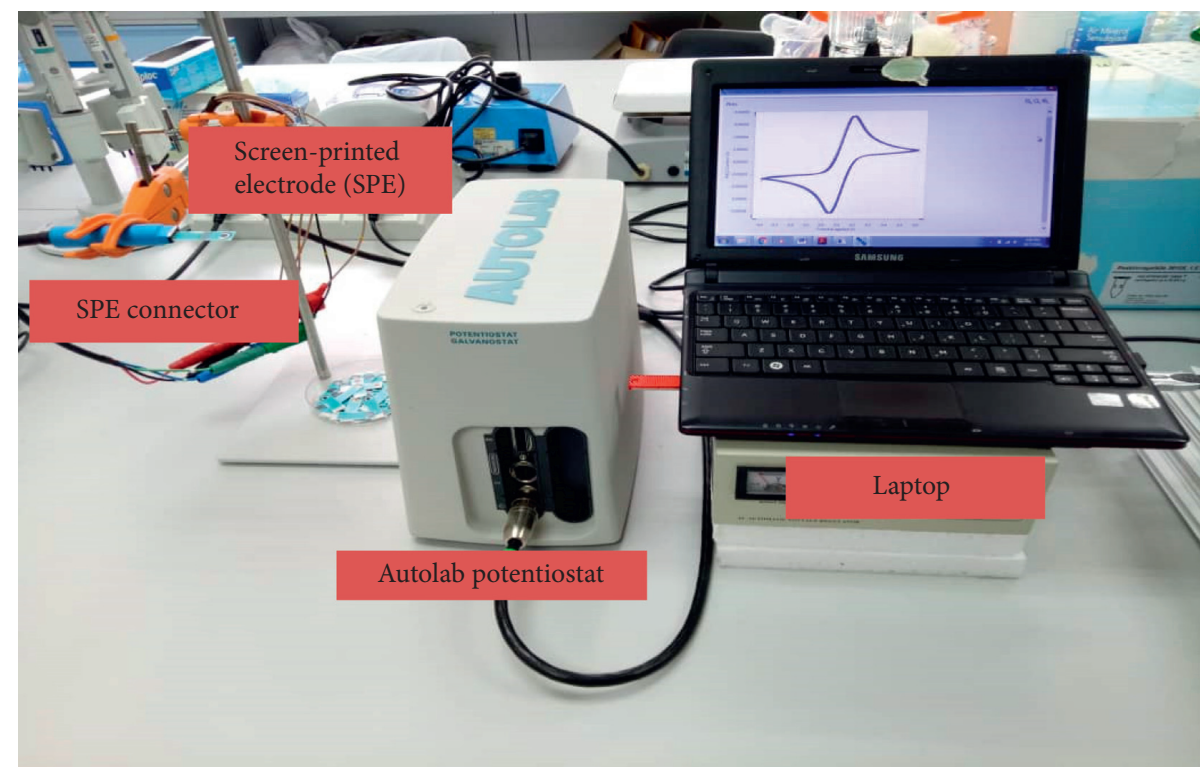

FIGURE 3: Schematic diagram of the electrochemical analysis setup using a screen-printed electrode (SPE).

aliphatic $\mathrm{C}-\mathrm{H}$ bonds observed with the pyrrole monomer at $2942 \mathrm{~cm}^{-1}[31,32]$. The wavenumber of stretching vibration for $\mathrm{C}=\mathrm{C}$ bonds was found slightly shifted from $1530 \mathrm{~cm}^{-1}$ (for pyrrole) to $1538 \mathrm{~cm}^{-1}$ for the PPy NPs rings, and the peak was noted slightly broader [33] while a sharp peak of stretching vibration $\mathrm{C}-\mathrm{N}$ bonds of pyrrole was observed at $1469-1417 \mathrm{~cm}^{-1}$ while deformation C-N bonds peaks of PPy NPs slightly shifted to a lower region at $1431-1409 \mathrm{~cm}^{-1}$ [13]. Furthermore, the C-N stretching bands of pyrrole at $1075 \mathrm{~cm}^{-1}$ and $1047 \mathrm{~cm}^{-1}$ were found to disappear from the PPy FTIR spectra $[9,30]$. The $\mathrm{C}-\mathrm{H}$ band in pyrrole appeared at $1013 \mathrm{~cm}^{-1}$ and was slightly shifted towards the lower region for the PPy NPs peak at $1003 \mathrm{~cm}^{-1}$ due to the out-of-plane bending $\mathrm{C}-\mathrm{H}$ bond $[31,34-36]$. The shift of these vibration bands indicates that the PPy NPs have been successfully produced.

3.2. Fourier Transform Infrared Spectroscopy of PolypyrroleIron Oxyhydroxide Nanocomposite. The characteristic band regions of organometallic compounds (500 to $2000 \mathrm{~cm}^{-1}$ ) are the main focus for observation of the interaction between $\mathrm{FeOOH}$ and PPy. A sharp peak of the $\mathrm{FeOOH}$ vibrational band was found at $691 \mathrm{~cm}^{-1}$ as shown in Figure 5(b). This observation is in concordance with the research reported by Wei et al. [37]. Fe-O vibrational modes in $\mathrm{FeOOH}$ were ascribed at 1640, 1363, and $1141 \mathrm{~cm}^{-1}$ in the PFFs spectra while there were no peaks in 


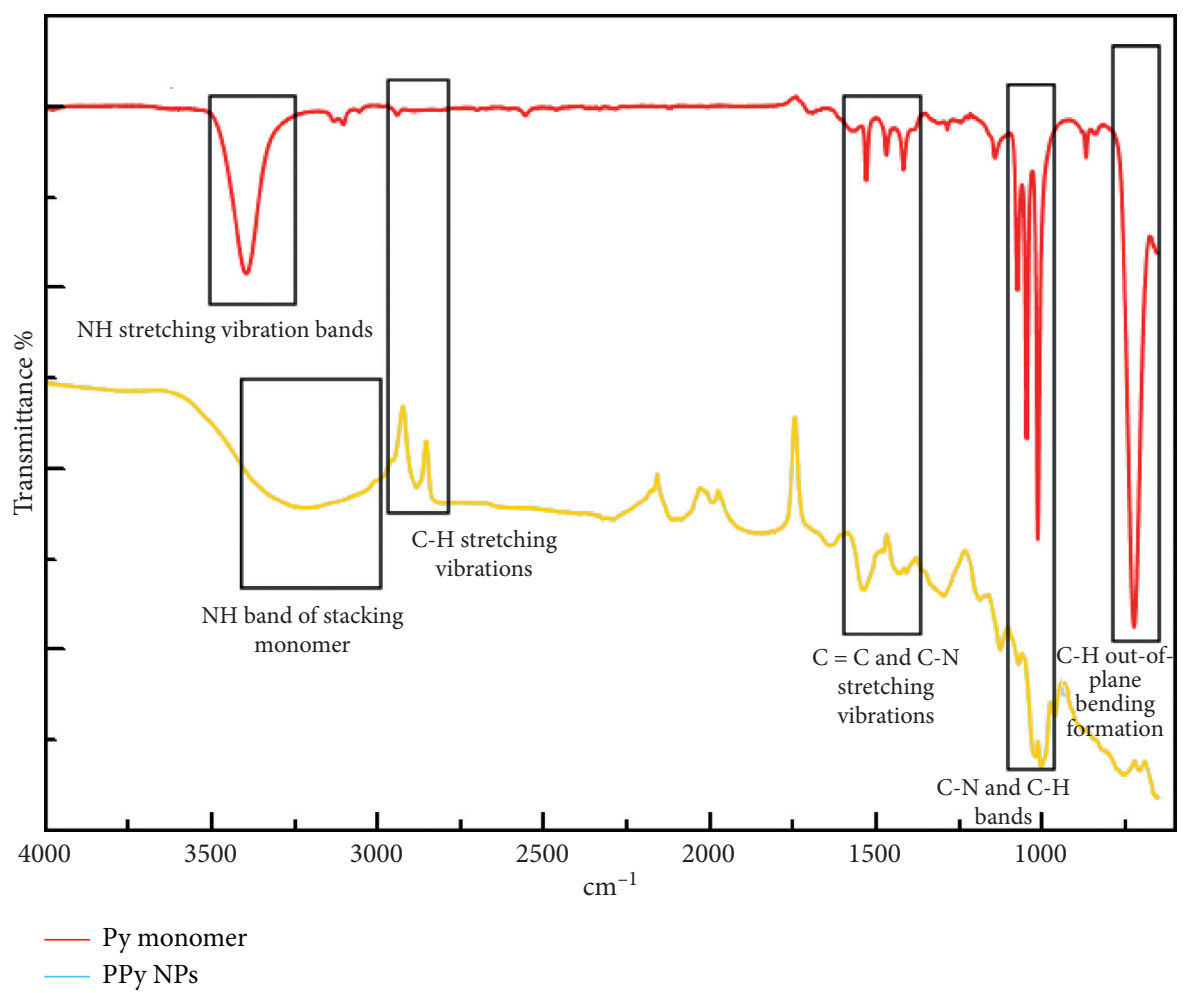

FIGURE 4: FTIR spectra of pyrrole monomer and PPy NPs.

the PPy NPs spectra [38]. Furthermore, the presence of $-\mathrm{OH}$ vibrational modes also could be detected at $891 \mathrm{~cm}^{-1}$ which is assigned as $-\mathrm{OH}$ bending modes and sharp -OH stretching mode at $3300 \mathrm{~cm}^{-1}[39,40]$. In the PPy NPs, this region $\left(3217 \mathrm{~cm}^{-1}\right)$ is assigned as the secondary amine band $(-\mathrm{NH})$. However, when the $\mathrm{FeOOH}$ attaches to the PPy NPs at the N atom (as shown in the schematic diagram in Figure 6), this secondary amine has changed to become a tertiary amine. The tertiary amine does not show any band in this region [41]. Therefore, this indicates that the peak observed in this region as illustrated in Figure 5(a) belongs to the $-\mathrm{OH}$ stretching band from $\mathrm{FeOOH}[40]$. These changes and the additional peaks confirm that the PFFs nanocomposite was produced. Figure 6 illustrates the schematic interaction of PFFs nanocomposite.

\subsection{Field Emission Scanning Electron Microscopy (FESEM) of} Polypyrrole Nanoparticles and Polypyrrole-Iron Oxyhydroxide Nanocomposite. The morphology and surface conditions of samples can be observed using FESEM imagery. Figure 7(a) shows the synthesised PPy NPs that did not undergo the sonication process. From the figure, it can be seen that the morphology of PPy is agglomerated and looks like linked globular subparticles, as suggested by Effati et al. [42]. Figure 7(b) shows the SEM images for the PPy that underwent the sonication process for one hour. The improvement of PPy NPs morphology can be observed by the disappearance of "linked globular subparticles" and the nanoparticles can now be clearly seen. However, the shape of the nanoparticle is irregular and shows a rough surface, which is commonly described as cauliflower morphology [43]. The morphology of PPy NPs with a longer time of sonication (three hours) is shown in Figure 7(c). It can be seen that the PPy NPs have turned back to become an agglomerate and show a clump and globular structure [40]. This indicates that a one-hour sonication time is enough to produce fine nanoparticles of PPy. The size of the nanoparticle obtained will be discussed in the TEM section as follows.

The preparation of PFFs was done by the combination of prepared PPy NPs with iron (III) chloride $\left(\mathrm{FeCl}_{3}\right)$ and sodium hydroxide $(\mathrm{NaOH})$. The concentration of $\mathrm{FeCl}_{3}$ was varied into $5 \%$ and $10 \mathrm{wt} \%$ in each preparation. The morphology of PFFs using $5 \mathrm{wt} \%$ of $\mathrm{FeCl}_{3}$ indicates spherical shapes as shown in Figure 7(d). The shape of the PFFs nanocomposite is better defined compared to the PPy NPs, thus leading to the formation of a porous PPy NPs shell with FeOOH NPs. This observation has also been reported by Guo et al. [44] after a long time of stirring (seven hours). In our finding, we were able to reduce the stirring time to only two hours together with one-hour duration of ultrasonication. The morphology of $10 \mathrm{wt} \%$ of $\mathrm{FeCl}_{3}$ is depicted in Figure 7(d). It shows that, with a higher concentration of $\mathrm{FeCl}_{3}$, the $\mathrm{FeOOH}$ was covered with a spherical shape of PPy particles that resulted in multiple agglomerated particles. This finding is in concordance with the research reported by Nalage et al. [45], who showed that agglomeration tends to occur with an increasing concentration of metal ion together with the PPy. 


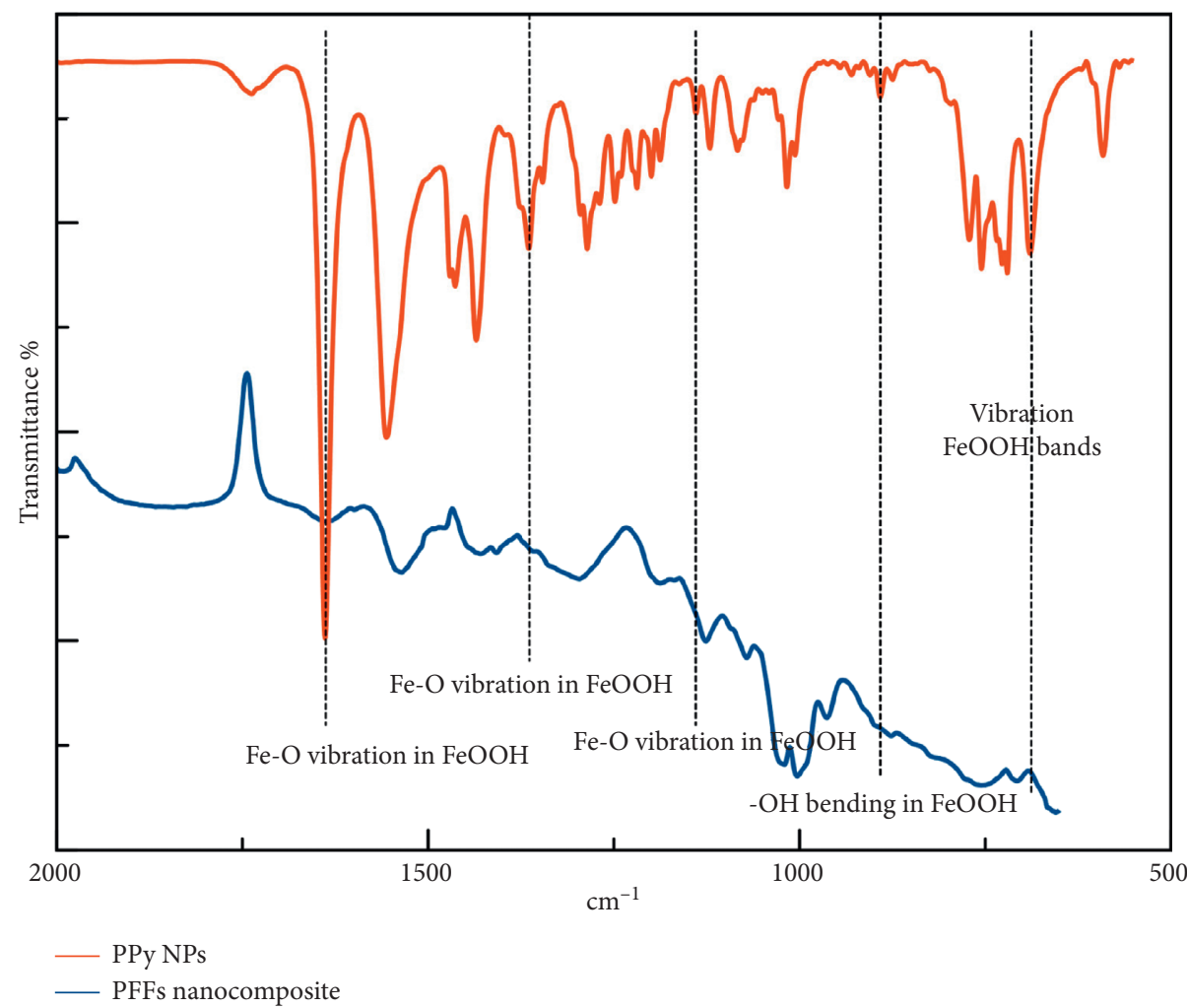

(a)

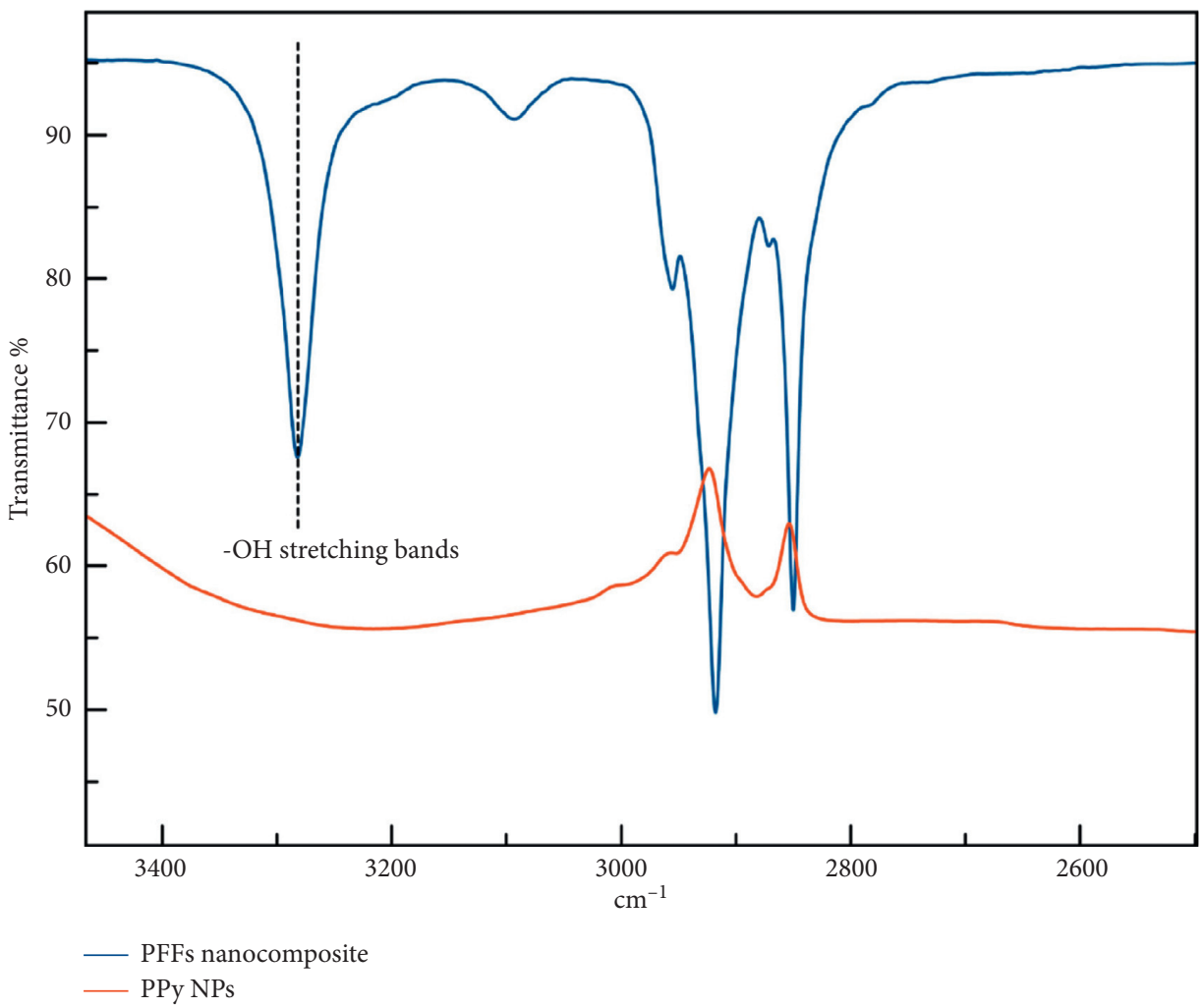

(b)

FIGURE 5: FTIR spectra of PPy NPs and PFFs nanocomposite at $2000-3500 \mathrm{~cm}^{-1}$ (a) and at $500-2000 \mathrm{~cm}^{-1}$ (b). 


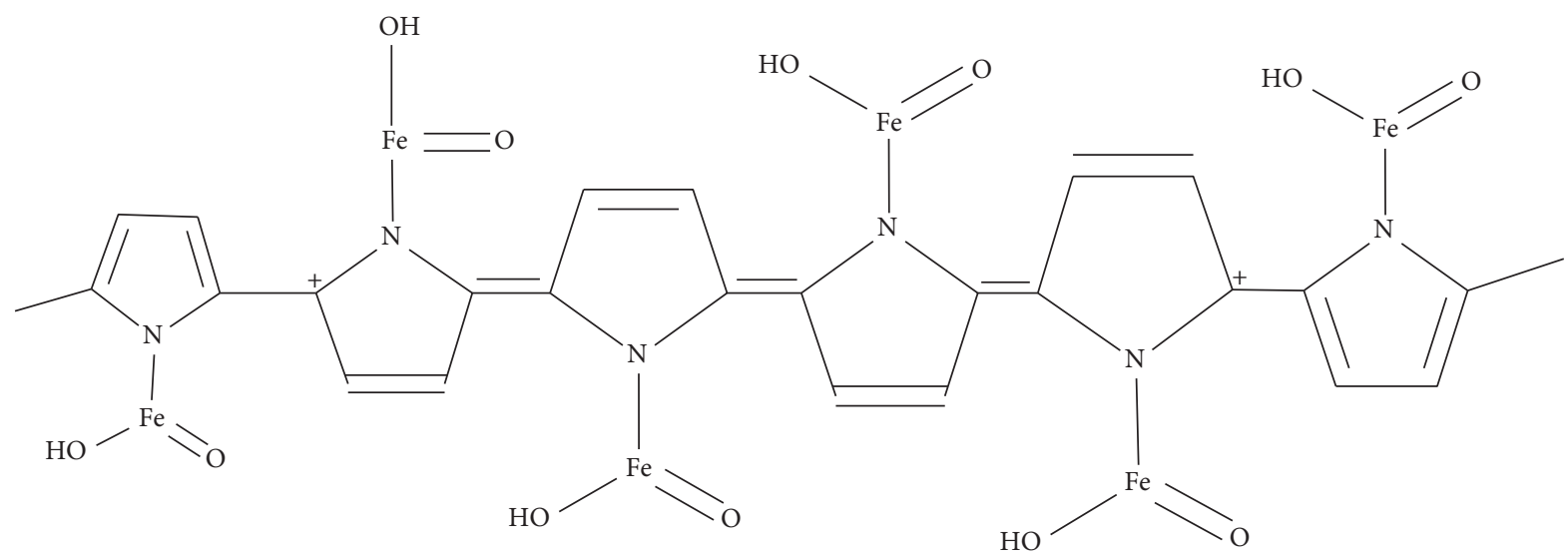

FIGURE 6: Suggested schematic diagram of PFFs nanocomposite in the detection of DMMP.

3.4. Transmission Electron Microscopy of Polypyrrole Nanoparticles and Polypyrrole-Iron Oxyhydroxide Nanocomposite. This analysis was performed to observe the size range of prepared PPy NPs and PFFs nanocomposite and to identify if the polymer obtained is within nanoscale parameters. Figure 8(a) depicts the TEM image of the PPy NPs after onehour sonication. It shows that the particle size range obtained was between 50 and $70 \mathrm{~nm}$ (with the average size of $67.18 \pm 12.69$ ), which is within the size parameters to be classified as nanoparticles, and this finding corroborates with other works which also prepared PPy NPs [46, 47]. In addition, these nanoparticles show linear structural shapes from the polymerisation process as described by Ahn et al. [48] while on the other hand, some of the spherical-shaped particles seen connected between the head and tail of linear PPy as seen in the image were due to irreversible aggregations of particles [45]. Figure 8(b) shows the TEM image of PFFs nanocomposite. The size of this nanocomposite was ranged between 110 and $160 \mathrm{~nm}$ (with the average size of $138.6 \pm 26.60)$ which is larger than the size of the PPy nanoparticles due to the presence of a higher insulating $\mathrm{FeOOH}$ particle loaded within the nanocomposite as has been reported by Guo et al. [44]. This can be proved that the sizes of PPy NPs are much smaller compared to PFFs as stated in FESEM.

3.5. Electrochemical Properties of Polypyrrole-Iron Oxyhydroxide Nanocomposite in Ferricyanide Solution. Cyclic voltammetry $(\mathrm{CV})$ is a concise process for measuring the formal potential of half-reactions when both the oxidised and reduced forms are stable during the time required to obtain a current-potential graph that is commonly known as a voltammogram [49]. A screen-printed carbon electrode (SPCE) is chosen as the test electrode with the addition of PPy NPs and PFFs nanocomposite for the detection of the analyte DMMP $(10 \mu \mathrm{L})$. SPCEs are commonly used as sensor constructs as they are easy to use and are cost-efficient in terms of their fabrication [50]. Ferricyanide $\left[\mathrm{Fe}(\mathrm{CN})_{6}\right]^{3-}$ is used as the redox probe to observe the electron transfer reaction which reflects the performance of the modified electrode [51]. Figure 9(a) represents the comparative $\mathrm{CV}$ of different types of modified electrode surfaces, specifically bare electrodes, PPy NPs, and PFFs nanocomposite with concentrations of either 5 or $10 \mathrm{wt} \%$ in $5 \mathrm{mmol}$ of $\left[\mathrm{Fe}(\mathrm{CN})_{6}\right]^{3-}$ in $0.1 \mathrm{M}$ of $\mathrm{KCl}$ solution. It can be seen that the oxidation peak (current $\sim 57.24 \mu \mathrm{A}$ and potential $\sim 0.22 \mathrm{~V}$ ) for the bare SPCE with a peak separation of $\left(\Delta E_{\mathrm{p}}\right) \sim 240 \mathrm{mV}$ was obtained. Then, the SPCE modified with PPy showed an enhancement in the oxidation peak current $\sim 75.88 \mu \mathrm{A}$ with $\Delta \mathrm{Ep} \sim 170 \mathrm{mV}$ which was higher than the bare SPCE. However, the SPCE with PFFs of 5 and $10 \mathrm{wt} \%$ showed a decrement in their oxidation peak currents, namely, $\sim 70.07 \mu \mathrm{A}\left(\Delta \mathrm{E}^{\mathrm{P}} \sim 166\right.$ $\mathrm{mV})$ and $69.16 \mu \mathrm{A}\left(\Delta \mathrm{E}_{\mathrm{p}} \sim 190 \mathrm{mV}\right)$, respectively.. However, the $10 \mathrm{wt} \%$ showed $69.16 \mu \mathrm{A}$ and $\Delta E_{\mathrm{p}} \sim 190 \mathrm{mV}$, respectively. The increase of current signal by $32.6 \%$ for PPy NPs that is higher than only $22.4 \%$ for the PFFs nanocomposite is due to the improvement of surface area, which has been confirmed by the TEM analysis. According to Liang et al. [52], the decrease in the oxidation peak current for the PFFs nanocomposite, both 5 and $10 \mathrm{wt} \%$, appears to be related to the presence of $\mathrm{FeOOH}$ molecules that have relatively high resistance and weak cyclic stability. Furthermore, the SPCE modified with PPy NPs $(\sim 170 \mathrm{mV})$ appeared to demonstrate lower peak separation values compared to the bare electrode as well as the other modified electrode due to an increase in the contact surface area between the nanostructure of PPy and the electrolyte $\left[\mathrm{Fe}(\mathrm{CN})_{6}\right]^{3-}$ as reported by Ahn et al. [48].

3.6. Electrochemical Behaviour of Polypyrrole-Iron Oxyhydroxide Nanocomposite (PFFs) towards DMMP. In order to apply the PPy NPs and PFFs nanocomposite as sensing materials for the detection of DMMP, the electrochemical behaviour of the PFFs nanocomposite was investigated. Figure 9(b) shows the CV of PPy NPs and PFFs nanocomposite-modified SPCE, with and without DMMP, respectively. It can be seen that the presence of DMMP affects the current scan for both PPy NPs and PFFs nanocomposite-modified SPCE compared to the modified electrodes without DMMP exposure. Figure 9(c) summarises the oxidation peak currents for PPy NPs and PFFs 


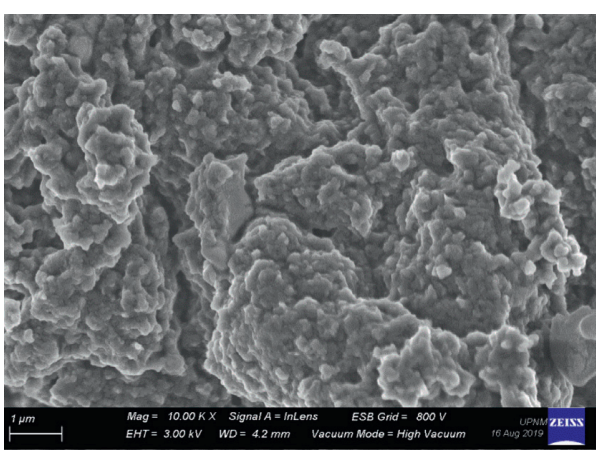

(a)

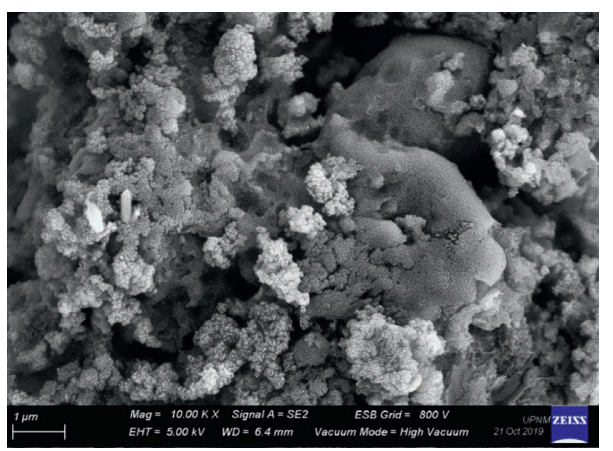

(c)

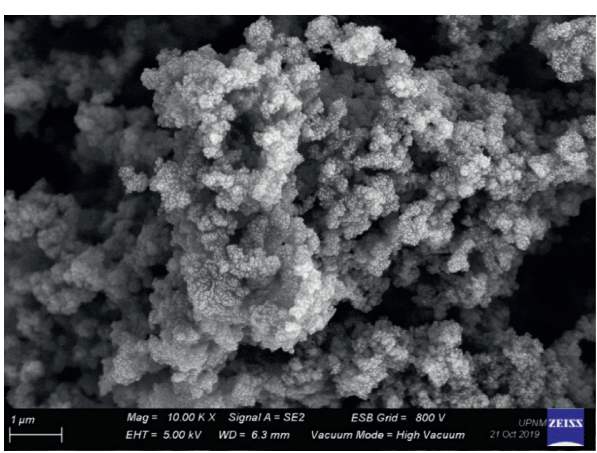

(b)

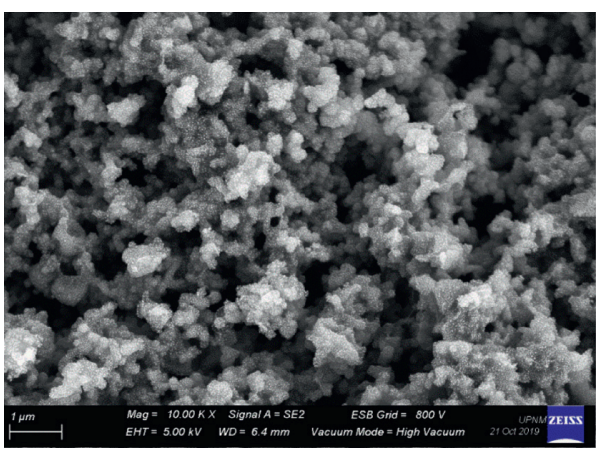

(d)

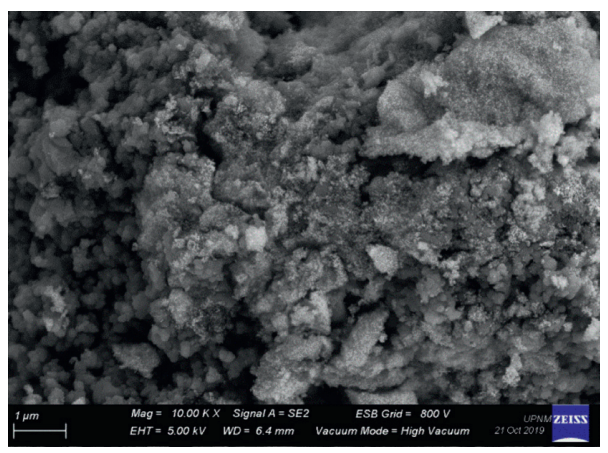

(e)

FIgURE 7: FESEM images with no sonication (a), one-hour sonication (b), three-hour sonication (c), 5 wt\% PFFs (d), and 10 wt\% PFFs (e).

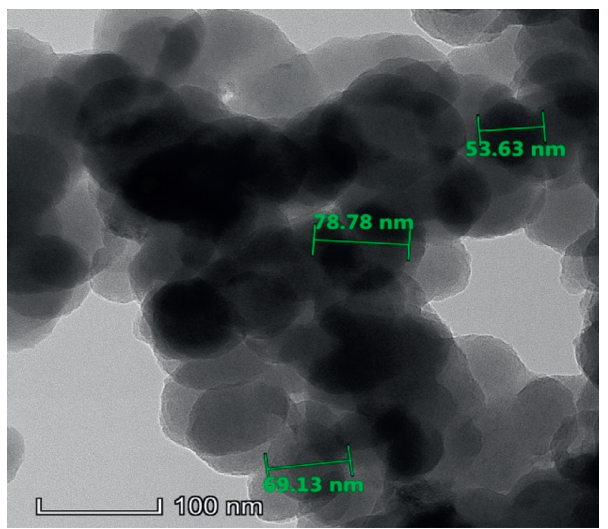

(a)

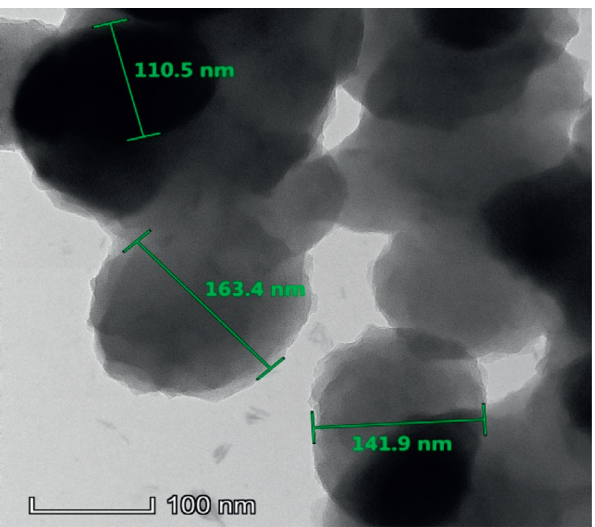

(b)

Figure 8: TEM images of PPy NPs (a) and 5 wt\% PFFs nanocomposite (b). 


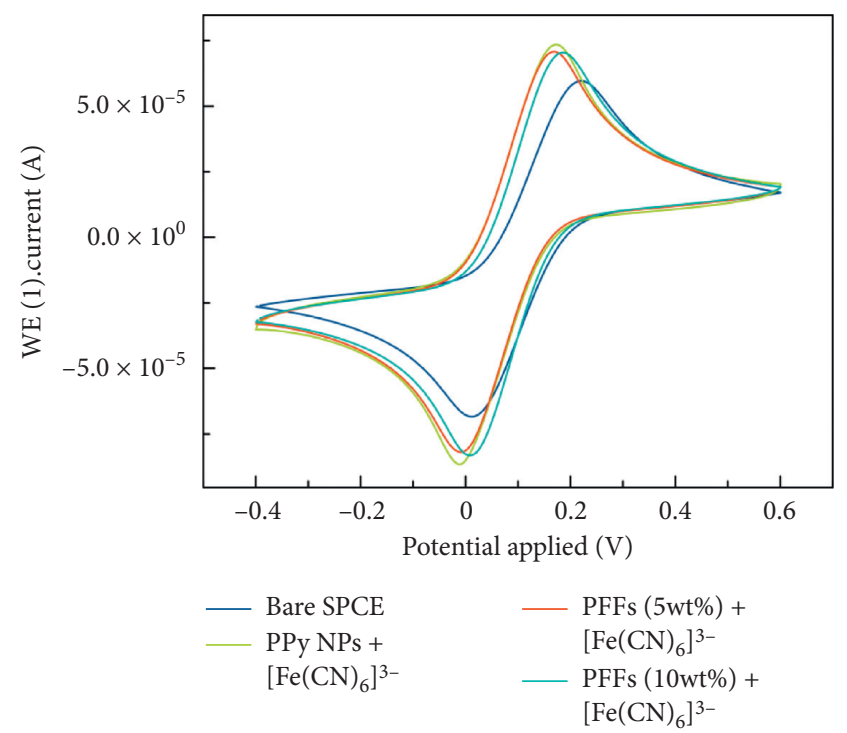

(a)

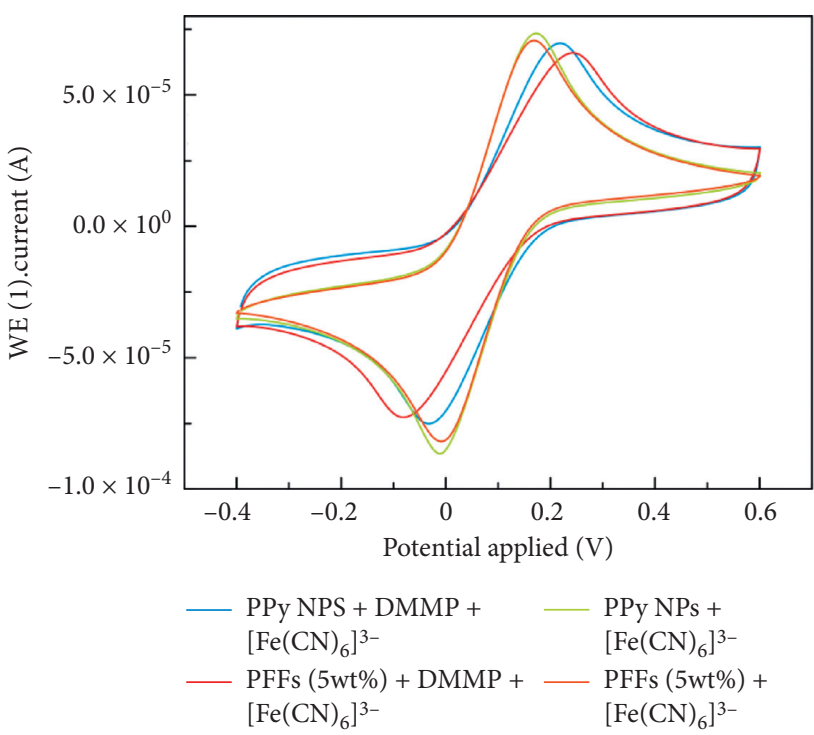

(b)

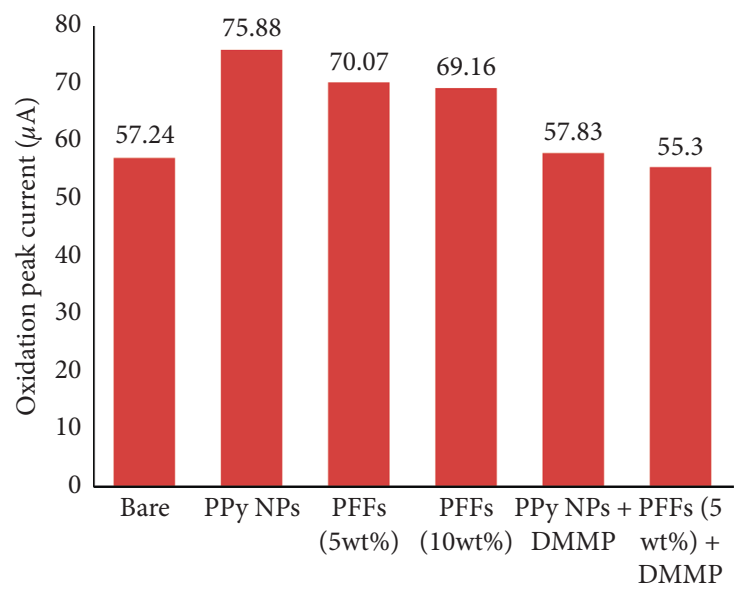

System

(c)

Figure 9: Cyclic voltammogram of bare electrode, PPy NPs, and $5 \mathrm{wt} \%$ and $10 \mathrm{wt} \%$ of PFFs without DMMP (a), PPy NPs and PFFs 5 wt $\%$ with DMMP (b), and oxidation peak current with different sensing materials on the surface of SPCE; PPy NPs, PFFs (5 wt\%), PFFs (10 wt\%), PPy NPs + DMMP, and PFFs (5 wt\%) + DMMP (c).

nanocomposite-modified electrodes exposed to DMMP. The oxidation peak current for PPy NPs $(\sim 75.88 \mu \mathrm{A})$ decreased to $\sim 57.83 \mu \mathrm{A}$ while the PFFs nanocomposite peak current decreased from $\sim 70.07 \mu \mathrm{A}$ to $\sim 55.30 \mu \mathrm{A}$ in the presence of DMMP. Moreover, peak separation values $\left(\Delta E_{\mathrm{p}}\right)$ for PPy NPs and PFFs nanocomposite-modified electrodes when exposed to DMMP were also observed (Figure 9(b)). The peak separation for the SPCE with PPy NPs was significantly lower $\left(\Delta E_{\mathrm{p}} \sim 230\right)$ compared to the PFFs nanocomposite-modified SPCE $\left(\Delta E_{\mathrm{p}} \sim 310\right)$. The reduction of the oxidation peak and increment of peak separation was due to a longer electron path where the electrons need to move from DMMP towards $\mathrm{FeOOH}$ before being transferred to PPy NPs [42]. Both the PPy NPs and PFFs nanocomposite contribute towards the enhancement of surface contact between the electrode and electrolyte. Thus, it shortens the path for ion diffusion and access to rich accessible redox reaction sites [53]. The sensitivity in detecting the analyte DMMP is improved as the vertically aligned FeOOH on PPy NPs directly increases the DMMP active sensing sites [28].

\section{Conclusion}

PPy NPs and PFFs nanocomposite were successfully synthesised using a chemical oxidative polymerisation technique with two different periods of sonication time (one and three hours). The one-hour sonication time produced finer nanoparticles compared to three hours of sonication. The polymerisation of the pyrrole monomer was confirmed by the broadening of the sharp peak of $-\mathrm{NH}$ stretching at $3217 \mathrm{~cm}^{-1}$ while the interaction between $\mathrm{FeOOH}$ and PPy 
NPs was seen through the disappearance of the N-H band and the existence of a new - $\mathrm{OH}$ sharp band at around $3500 \mathrm{~cm}^{-1}$. FESEM imagery showed that the $5 \mathrm{wt} \% \mathrm{FeOOH}$ nanocomposite possessed iron metals that were well distributed without agglomeration in the PPy NPs compared to the $10 \mathrm{wt} \% \mathrm{FeOOH}$. The size of the prepared PPy NPs and PFFs nanocomposite was between 50 and $70 \mathrm{~nm}$ and between 110 and $160 \mathrm{~nm}$, respectively. The potential of PPy NPs and PFFs nanocomposite as sensing materials in detecting DMMP was demonstrated using cyclic voltammetry $(\mathrm{CV})$. A reduction of oxidation peak current has been observed in the CV measurement when DMMP was introduced to both the PPy NPs and PFFs nanocompositemodified electrodes. Therefore, PPY NPs and PFFs nanocomposite can be promising sensing materials in the construction of an electrochemical sensor for the detection of DMMP. A further study is recommended in terms of determining the sensitivity of the electrochemical sensing system in detecting different analytes with varying concentration range. In addition, the PPy NPs and PFFs nanocomposite have the potential to be utilised as sensing materials in gas sensors.

\section{Data Availability}

The data used to support the study are available within the article.

\section{Conflicts of Interest}

The authors declare that they have no conflicts of interest.

\section{Acknowledgments}

The authors are grateful to the Ministry of Education Malaysia, Development Fund F0020 for funding via UPNM/ 2018/CHEMDEF/ST/5.

\section{References}

[1] W. S. Chow and W. L. Tham, "Effects of antistatic agent on the mechanical, morphological and antistatic properties of polypropylene/organo-montmorillonite nanocomposites," Express Polymer Letters, vol. 3, no. 2, pp. 116-125, 2009.

[2] T.-H. Le, Y. Kim, and H. Yoon, "Electrical and electrochemical properties of conducting polymers," Polymers, vol. 9, no. 4, p. 150, 2017.

[3] Y.-Z. Long, M.-M. Li, C. Gu et al., "Recent advances in synthesis, physical properties and applications of conducting polymer nanotubes and nanofibers," Progress in Polymer Science, vol. 36, no. 10, pp. 1415-1442, 2011.

[4] G. A. Snook, P. Kao, and A. S. Best, "Conducting-polymerbased supercapacitor devices and electrodes," Journal of Power Sources, vol. 196, no. 1, pp. 1-12, 2011.

[5] R. H. Santim, H. A. de Aquino, and J. A. Malmonge, "Fabrication of polypyrrole nanoparticles using microemulsion polymerization for diferent Py/APS/SDS molar ratios," Materials Science Forum, vol. 869, pp. 391-395, 2016.

[6] S. Dey and A. Kumar Kar, "Morphological and optical properties of polypyrrole nanoparticles synthesized by variation of monomer to oxidant ratio," Materials Today: Proceedings, vol. 18, pp. 1072-1076, 2019.

[7] A. Imani, G. Farzi, and A. Ltaief, "Facile synthesis and characterization of polypyrrole-multiwalled carbon nanotubes by in situ oxidative polymerization," International Nano Letters, vol. 3, no. 1, p. 52, 2013.

[8] S. H. Kim, S. H. Jang, S. W. Byun et al., "Electrical properties and EMI shielding characteristics of polypyrrole-nylon 6 composite fabrics," Journal of Applied Polymer Science, vol. 87, no. 12, pp. 1969-1974, 2003.

[9] A. Reung-U-Rai, A. Prom-Jun, W. Prissanaroon-Ouajai, and S. Ouajai, "Synthesis of highly conductive polypyrrole nanoparticles via microemulsion polymerization," Journal of Metals, Materials and Minerals, vol. 18, no. 2, pp. 27-31, 2008.

[10] S. Bahraeian, K. Abron, F. Pourjafarian, and R. A. Majid, "Study on synthesis of polypyrrole via chemical polymerization method," Advanced Materials Research, vol. 795, pp. 707-710, 2013.

[11] J. Bo, X. Luo, H. Huang, L. Li, W. Lai, and X. Yu, "Morphology-controlled fabrication of polypyrrole hydrogel for solid-state supercapacitor," Journal of Power Sources, vol. 407, pp. 105-111, 2018.

[12] C. R. Rao, R. Muthukannan, J. A. Jebin, T. Antony, and M. Vijayan, "Synthesis and properties of polypyrrole obtained from a new Fe (III) complex as oxidizing agent," Indian Journal of Chemistry, vol. 52A, no. 6, pp. 744-748, 2013.

[13] A. Yussuf, M. Al-Saleh, S. Al-Enezi, and G. Abraham, "Synthesis and characterization of conductive polypyrrole: the influence of the oxidants and monomer on the electrical, thermal, and morphological properties," International Journal of Polymer Science, vol. 2018, Article ID 4191747, 8 pages, 2018.

[14] H. Wang, T. Lin, and A. Kaynak, "Polypyrrole nanoparticles and dye absorption properties," Synthetic Metals, vol. 151, no. 2, pp. 136-140, 2005.

[15] C. Yang, X. Wang, Y. Wang, and P. Liu, "Polypyrrole nanoparticles with high dispersion stability via chemical oxidative polymerization in presence of an anionic-non-ionic bifunctional polymeric surfactant," Powder Technology, vol. 217, pp. 134-139, 2012.

[16] S. Y. Chin, T. K. Abdullah, and M. Mariatti, "One-step synthesis of conductive graphene/polyaniline nanocomposites using sodium dodecylbenzenesulfonate: preparation and properties," Journal of Materials Science: Materials in Electronics, vol. 28, no. 24, pp. 18418-18428, 2017.

[17] S. Xing and G. Zhao, "Stability and particle size of polypyrrole dispersion using sodium dodecylbenzenesulfonate as surfactant," E-Polymers, vol. 7, no. 1, 2007.

[18] L. A. Patil, A. R. Bari, M. D. Shinde, V. Deo, and M. P. Kaushik, "Detection of dimethyl methyl phosphonate-a simulant of sarin: the highly toxic chemical warfare-using platinum activated nanocrystalline $\mathrm{ZnO}$ thick films," Sensors and Actuators B: Chemical, vol. 161, no. 1, pp. 372-380, 2012.

[19] S. Royo, R. Martínez-Máñez, F. Sancenón, A. M. Costero, M. Parra, and S. Gil, "Chromogenic and fluorogenic reagents for chemical warfare nerve agents' detection," Chemical Communications, vol. 46, no. 46, pp. 4839-4847, 2007.

[20] F. N. Diauudin, J. I. A. Rashid, V. F. Knight et al., "A review of current advances in the detection of organophosphorus chemical warfare agents based biosensor approaches," Sensing and Bio-Sensing Research, vol. 26, Article ID 100305, 2019.

[21] Z. Pei, X. Ma, P. Ding, W. Zhang, Z. Luo, and G. Li, "Study of a QCM dimethyl methylphosphonate sensor based on a $\mathrm{ZnO}$ - 
modified nanowire-structured manganese dioxide film," Sensors, vol. 10, no. 9, pp. 8275-8290, 2010.

[22] S. Sundarrajan, A. R. Chandrasekaran, and S. Ramakrishna, "An update on nanomaterials-based textiles for protection and decontamination," Journal of the American Ceramic Society, vol. 93, no. 12, pp. 3955-3975, 2010.

[23] O. S. Kwon, C. S. Park, and S. J. Park, "Carboxylic acidfunctionalized conducting-polymer nanotubes as highly sensitive nerve-agent chemiresistors," Scientific Reports, vol. 6, Article ID 33724, 2016.

[24] D. C. Tiwari, R. Sharma, K. D. Vyas, M. Boopathi, V. V. Singh, and P. Pandey, "Electrochemical incorporation of copper phthalocyanine in conducting polypyrrole for the sensing of DMMP," Sensors and Actuators B: Chemical, vol. 151, no. 1, pp. 256-264, 2010.

[25] M. Das and S. Roy, "Polypyrrole and associated hybrid nanocomposites as chemiresistive gas sensors: a comprehensive review," Materials Science in Semiconductor Processing, vol. 121, Article ID 105332, 2021.

[26] K. Malook, H. Khan, and M. Shah, "Ammonia sensing behavior of polypyrrole-bimetallic oxide composites," Polymer Composites, pp. 1-6, 2020.

[27] M. Šetka, J. Drbohlavová, and J. Hubálek, "Nanostructured polypyrrole-based ammonia and volatile organic compound sensors," Sensors, vol. 17, no. 3, p. 562, 2017.

[28] J. S. Lee, D. H. Shin, J. Jun, and J. Jang, "Multidimensional polypyrrole/iron oxyhydroxide hybrid nanoparticles for chemical nerve gas agent sensing application," ACS Nano, vol. 7, no. 11, pp. 10139-10147, 2013.

[29] Y. Tao, E. Ju, J. Ren, and X. Qu, "Polypyrrole nanoparticles as promising enzyme mimics for sensitive hydrogen peroxide detection," Chemical Communications, vol. 50, no. 23, pp. 3030-3032, 2014.

[30] A. Dhillon, A. Kaur, A. K. Srivastava, and D. K. Avasthi, "Experimental investigations of semi-crystalline plasma polymerized polypyrrole for surface coating," Progress in Organic Coatings, vol. 69, no. 4, pp. 396-401, 2010.

[31] M. Kamal and A. Bhuiyan, "Structural and optical characterization of plasma polymerized pyrrole monolayer thin films," Advances in Optoelectronic Materials (AOM), vol. 1, no. 2, pp. 11-17, 2013

[32] L. Gómez, M. G. Olayo, and G. J. Cruz, "Effect of energy in the size of pyrrole-derived particles synthesized by plasma," Superficies Y Vacío, vol. 25, no. 2, pp. 88-91, 2012.

[33] A. Reung-U-Rai, A. Prom-Jun, W. Ouajai, and S. Ouajai, "Synthesis of highly conductive polypyrrole nanoparticles via microemulsion polymerization," Journal of the Minerals Metals \& Materials Society, vol. 18, pp. 27-31, 2008.

[34] E. Manahil, A. Zainal, and H. Hassan, "Nodar preparation and characterization of polypyrrole conjugated polymer in the presence of ionic surfactant," Journal of Biomedical Materials Research, vol. 6, p. 111, 2014.

[35] Z. Ahmad, M. A. Choudhary, A. Mehmood, R. Wakeel, T. Akhtar, and M. A. Rafiq, "Synthesis of polypyrrole nano/ microspheres using cobalt(III) as an oxidizing agent and its ammonia sensing behavior," Macromolecular Research, vol. 24, no. 7, pp. 596-601, 2016.

[36] I. Ibrahim, S. Yunus, and M. Hashim, "Relative performance of isopropylamine, pyrrole and pyridine as corrosion inhibitors for carbon steels in saline water at mildly elevated temperatures," International Journal of Scientific \& Engineering Research, vol. 4, no. 2, pp. 1-12, 2013.

[37] G. Wei, K. Du, X. Zhao et al., "Integrated FeOOH nanospindles with conductive polymer layer for high-performance supercapacitors," Journal of Alloys and Compounds, vol. 728, pp. 631-639, 2017.

[38] S. Choudhari, D. Bhattacharya, and J.-s. Yu, "1-dimensional porous $\alpha-\mathrm{Fe}_{2} \mathrm{O}_{3}$ nanorods as high performance electrode material for supercapacitor," RSC Adv, vol. 3, no. 47, Article ID 25120, 2013.

[39] L. Mei, L. Liao, Z. Wang, and C. Xu, "Interactions between phosphoric/tannic acid and different forms of $\mathrm{FeOOH}$," Advances in Materials Science and Engineering, vol. 2015, Article ID 250836, 10 pages, 2015.

[40] S. Kang, G. Wang, M. Fang, H. Wang, X. Wang, and W. Cai, "Water bath synthesis and enhanced photocatalytic performances of urchin-like micro/nanostructured $\alpha$-FeOOH," Journal of Materials Research, vol. 30, no. 10, pp. 1629-1638, 2015.

[41] R. A. Parildar and A. A. B. Ibik, "Characterization of tertiary amine and epoxy functional all-acrylic coating system," Progress in Organic Coatings, vol. 76, no. 6, pp. 955-958, 2013.

[42] E. Effati, B. Pourabbas, and M. S. Zakerhamidi, "Continuous microfluidic fabrication of polypyrrole nanoparticles," RSC Advances, vol. 9, no. 30, pp. 16977-16988, 2019.

[43] A. Olad and S. Shakoori, "Electromagnetic interference attenuation and shielding effect of quaternary epoxy-PPy/ $\mathrm{Fe}_{3} \mathrm{O}_{4}$ - $\mathrm{ZnO}$ nanocomposite as a broad band microwave-absorber," Journal of Magnetism and Magnetic Materials, vol. 458, pp. 335-345, 2018.

[44] Z. Guo, K. Shin, A. B. Karki, D. P. Young, R. B. Kaner, and H. T. Hahn, "Fabrication and characterization of iron oxide nanoparticles filled polypyrrole nanocomposites," Journal of Nanoparticle Research, vol. 11, no. 6, pp. 1441-1452, 2009.

[45] S. R. Nalage, A. T. Mane, R. C. Pawar, C. S. Lee, and V. B. Patil, "Polypyrrole-NiO hybrid nanocomposite films: highly selective, sensitive, and reproducible $\mathrm{NO}_{2}$ sensors," Ionics, vol. 20, no. 11, pp. 1607-1616, 2014.

[46] Q. Lü, "Unstirred preparation of soluble electroconductive polypyrrole nanoparticles," Microchimica Acta, vol. 168, no. 3-4, pp. 205-213, 2010.

[47] Y. Liu, Y. Chu, and L. Yang, "Adjusting the inner-structure of polypyrrole nanoparticles through microemulsion polymerization," Materials Chemistry and Physics, vol. 98, no. 2-3, pp. 304-308, 2006.

[48] K.-J. Ahn, Y. Lee, H. Choi et al., "Surfactant-templated synthesis of polypyrrole nanocages as redox mediators for efficient energy storage," Scientific Reports, vol. 5, Article ID 14097, 2015.

[49] P. T. Kissinger and W. R. Heineman, "Cyclic voltammetry," Journal of Chemical Education, vol. 60, no. 9, p. 702, 1983.

[50] Y. Ye and H. Ju, "Rapid detection of ssDNA and RNA using multi-walled carbon nanotubes modified screen-printed carbon electrode," Biosensors and Bioelectronics, vol. 21, no. 5, pp. 735-741, 2005.

[51] V. Ganesh and V. Lakshminarayanan, "Microemulsion phase as a medium for electrodeposition of nickel and electrontransfer study of ferrocyanide-ferricyanide redox system," Journal of Colloid and Interface Science, vol. 349, no. 1, pp. 300-306, 2010.

[52] W. Liang, R. Poon, and I. Zhitomirsky, "Zn-doped FeOOHpolypyrrole electrodes for supercapacitors," Materials Letters, vol. 255, Article ID 126542, 2019.

[53] A. Karimi, I. Kazeminezhad, L. Naderi, and S. Shahrokhian, "Construction of a ternary nanocomposite, polypyrrole/FeCo sulfide-reduced graphene oxide/nickel foam, as a novel binder-free electrode for high-performance asymmetric supercapacitors," The Journal of Physical Chemistry C, vol. 124, no. 8, pp. 4393-4407, 2020. 